\title{
APRENDIZAJE A LO LARGO DE LA VIDA Y EDUCACIÓN SUPERIOR DE ADULTOS EN EL ÁMBITO UNIVERSITARIO EN ALEMANIA. ORIGEN Y EVOLUCIÓN HISTÓRICA HASTA LA ACTUALIDAD
}

\section{LIFELONG LEARNING AND ADULT HIGHER EDUCATION IN THE UNIVERSITARY FIELD IN GERMANY. ORIGIN AND HISTORICAL DEVELOPMENT UNTIL THE PRESENT}

Alicia Sianes-Bautista

Universidad de Sevilla

\section{RESUMEN}

Para el presente estudio se ha trabajado a partir de dos conceptos, en múltiples ocasiones empleados como sinónimos y sobre los que convendría, manifiestamente, establecer claras diferencias, a saber: el Aprendizaje a lo largo de la Vida y la Educación de Adultos. En una primera parte, se exponen definiciones aportadas por diversos autores e instituciones de renombre como la OCDE o la UNESCO para los conceptos objeto de estudio atendiendo, con especial interés, al origen de los mismos además de a su perspectiva histórica. No obstante, en una parte posterior, se torna la mirada hacia el papel que han desempeñado el Aprendizaje a lo Largo de la Vida y la Educación de Adultos a lo largo de la historia hasta la actualidad, en un nivel académico determinado, en este caso la Educación Superior Universitaria y en Alemania. Los resultados obtenidos reflejan, en primer lugar, la juventud que caracteriza a estos conceptos, acuñándose en el Siglo XIX; por otro lado, se hace alusión al desacuerdo entre países a la hora de definir estos conceptos debido a discrepancias entre factores tales como la edad, el nivel educativo, la formalidad del aprendizaje...; en la actualidad, las diferencias se hacen más notorias, enfocándose el Aprendizaje a lo largo de la Vida hacia la Educación Permanente y, la Educación Superior de Adultos en el ámbito universitario, hacia vías de Acceso a la Universidad.

Palabras clave: Aprendizaje a lo largo de la Vida, Educación de Adultos, Universidad, Alemania, Evolución histórica.

\section{ABSTRACT}

This study focuses on two concepts: Lifelong Learning and Adult Education. These concepts are frequently used as synonyms, thus creating the need to establish clear differences between them. Firstly, several definitions of these concepts are 
provided from many renowned authors and institutions like the OECD or UNESCO, based on their origin and historical perspective. Next, this study analyses the role played by Lifelong Learning and Adult Education throughout history until the present, with a focus on the University Higher Education in Germany. The results highlight the newness of these concepts which were coined in the 19th Century as well as the lack of a common definition among different countries due to variances such as age, academic level, formality of the learning process and other factors. More recently, the differences have become even more significant, focusing Lifelong Learning on Permanent Education and Higher Adult Education on University Access processes.

Keywords: Lifelong Learning, Adult Education, University, Germany, Historical evolution.

\section{INTRODUCCIÓN}

La Educación de las Personas Adultas es considerada como un campo específico de la educación muy reciente y, específicamente, la Educación de Adultos tal y como se conoce hoy en día lo es aún más (Tiana Ferrer, 1991). A partir de finales del Siglo XVIII tienen lugar dos acontecimientos de gran importancia en Europa: en primer lugar la expansión de la Revolución Industrial y, en segundo lugar la desaparición de la sociedad estamental característica del Antiguo Régimen. Convirtiéndose así el Siglo XIX en aquel en el que tienen lugar las primeras propuestas y avances que desembocan en la Educación de Adultos propiamente dicha.

Para hacer referencia a un término, es necesario tener constancia de a qué se está aludiendo. No obstante, en lo que a la definición de Educación de Adultos se refiere, la UNESCO reconoce en el año 1953 que, por el momento se carece de unidad o consenso alguno en las definiciones de Educación de Adultos en los diversos países que a la misma hacen referencia. Y no fue hasta el año 1976 cuando fue aprobada por la UNESCO una definición que era más aplicable a la experiencia relacionada con el término en cuestión y resulta de interés para la delimitación de su campo histórico, pues posee criterios que conviene tener en cuenta, entre los cuales destacan:

$\checkmark$ En primer lugar, la Educación de Adultos abarca un extenso conjunto de procesos educativos cuyo carácter es sistemático. Lo cual implica la aprobación de las prácticas que posean un mínimo carácter organizado, suprimiéndose así las que carezcan del mismo. 
$\checkmark$ En segundo lugar se hace alusión a los beneficiarios de este tipo de educación, los cuales son, según la UNESCO, aquellas personas consideradas adultas por la sociedad a la que pertenecen. Con esto se quiere decir que normalmente este tipo de educación ha estado principalmente dirigida a personas en edad postescolar, independientemente de la obligatoriedad de la misma.

$\checkmark$ La UNESCO admite que se incluyan actividades cuyo carácter sea formal, no formal e informal.

$\checkmark$ Se acepta asimismo por parte de la UNESCO la diversidad interna del campo de referencia, es decir, que permite englobar bajo la denominación de Educación de Adultos tanto cursos de alfabetización primaria, como cursos de formación profesional, actividades recreativas, prácticas de extensión universitaria, etc.

Tiana Ferrer (1991:10) en su trabajo acepta como educación de adultos:

La ofrecida a personas que habían superado la edad escolar habitual (fuese obligatoria 0 no) y abandonado el sistema educativo formal, aunque su consideración de "personas adultas" sea estrictamente discutible. De no hacerlo así, excluiríamos un amplio conjunto de actividades cuyos principales destinatarios fueron jóvenes, aunque prematuramente integrados en el mundo laboral y adulto.

Tomando la definición anterior como punto de partida, en el presente trabajo se pretende dirigir la Educación de Adultos hacia aquella de carácter formal, especialmente enfocándola al ámbito universitario. Por ello se presenta a continuación la definición de educación formal de adultos ('Formal Adult Education') proporcionada por EURYDICE (2011:17) donde se mantiene que la educación formal de adultos ha sido comúnmente entendida para referirse a actividades de aprendizaje encauzadas a la obtención de diplomas o certificados equivalentes a los obtenidos en la escuela $u$ otra clase de sistemas de Educación Superior ${ }^{1}$.

Asimismo, si se pretende hacer alusión al término "educación formal" resulta apropiado distinguir entre la educación formal, la educación no formal y la educación informal, para lo cual se ha recurrido al manual del Eurosat Classification of Learning Activities (CLA) en el que la educación formal se define como:

\footnotetext{
1 'Formal adult education' in commonly understood to mean learning activities leading to diplomas and certificates equivalent to those which may be obtained in the school or higher education system.
} 
Educación proporcionada en el sistema de escuelas, universidades y otras instituciones educativas formales que normalmente constituyen una "escalera" de educación continua a tiempo completo para niños y jóvenes que generalmente comienza de los cinco a los siete años y continua hasta los 20 o los 25 años. En algunos países, las partes superiores de esta "escalera" son programas organizados en los que se combinan el empleo y la participación en el sistema escolar y universitario ordinario a tiempo parcial: estos programas han llegado a conocerse como "sistema dual" o términos equivalentes en estos países". (Eurosat 2006:13)².

\section{La educación no formal se define como:}

Cualquier actividad educativa organizada y sostenida que no se corresponda exactamente con la definición anterior de educación formal. La educación no formal puede tener lugar tanto dentro como fuera de las instituciones educativas y se destina a personas de todas las edades. Dependiendo de los contextos de los países, puede abarcar programas educativos en los que se imparta alfabetización de adultos, educación básica para niños no escolarizados, formación práctica, capacitación laboral y cultura general. Los programas de educación no formal no necesariamente siguen el sistema de "escalera" y pueden tener una duración diferente". (Eurosat, 2006:13)³.

La educación informal, es:

Intencional, pero está menos organizada y menos estructurada... y puede incluir, por ejemplo, eventos (actividades) de aprendizaje que se producen en la familia, en el lugar de trabajo en la vida cotidiana de cada persona que aprende, de forma auto-dirigida, dirigida por la familia o la sociedad" (Eurosat, 2006:13) 4 .

\footnotetext{
2 Definición original, formal education: "...education provided in the system of schools, colleges, universities and other formal educational institutions that normally constitutes a continuous "ladder" of full-time education for children and young people, generally beginning at age of fi ve to seven and continuing up to 20 or 25 years old. In some countries, the upper parts of this "ladder" are organised programmes of joint part-time employment and part-time participation in the regular school and university system: such programmes have come to be known as the "dual system" or equivalent terms in these countries."

3 Definición original, non-formal education: "any organised and sustained educational activities that do not correspond exactly to the above definition of formal education. Non-formal education may therefore take place both within and outside educational institutions, and cater to persons of all ages. Depending on country contexts, it may cover educational programmes to impart adult literacy, basic education for out of school children, life-skills, work-skills, and general culture. Non formal education programmes do not necessarily follow the "ladder" system, and may have a differing duration".

4 Definición original, informal education: "...intentional, but it is less organised and less structured .... and may include for example learning events (activities) that occur in the family, in the work place, and in the daily life of every person, on a self-directed, familydirected or socially directed basis".
} 
El aprendizaje es el producto esencial que se obtiene de la educación, por lo tanto si se ha hecho distinción previamente entre los tipos de educación, no sería descabellado hacer una diferenciación entre los tipos de aprendizaje, entre los que se encuentran el aprendizaje formal, el aprendizaje no formal y el aprendizaje informal. Según el glosario de la Cedefop en el año 2008, el aprendizaje formal se define como:

\begin{abstract}
Aprendizaje que tiene lugar en entornos organizados y estructurados (p.e. un centro educativo o formativo, o bien en el centro de trabajo) y que se designa explícitamente como formación (en cuanto a sus objetivos, su duración y los recursos empleados). La formación o aprendizaje formal presupone intencionalidad por parte del alumno. Por regla general, siempre da lugar a una validación y una certificación o titulación". (Cedefop 2008:85).
\end{abstract}

El aprendizaje no formal se define como:

Aprendizaje derivado de actividades planificadas pero no designadas explícitamente como programa de formación (en cuanto a objetivos didácticos, duración o soportes formativos). El aprendizaje no formal presupone intencionalidad por parte del alumno. (Cedefop, 2008:134).

El aprendizaje informal se define como:

Aprendizaje resultante de actividades cotidianas relacionadas con el trabajo, la vida familiar o el ocio. No se halla organizada ni estructurada en cuanto a sus objetivos, duración o recursos formativos. Los aprendizajes informales carecen por regla general de intencionalidad por parte del alumno. (Cedefop, 2008:93).

\title{
2. APRENDIZAJE A LO LARGO DE LA VIDA Y EDUCACIÓN DE ADULTOS
}

El Aprendizaje a lo largo de la Vida, concepto ampliamente utilizado por el discurso anglosajón -Lifelong Learning-constituye un paradigma que presenta cierta complejidad en lo que a su terminología y conceptualización se refiere. Autores como Torres hacen alusión a múltiples términos que han sido empleados, en discursos políticos y debates académicos (Educación Continua, Educación de Adultos, Educación para Todos, Educación Permanente, Formación a lo largo de la Vida, etc.) (Torres, 2003 en García Ruíz, 2005).

Las reformas relacionadas con este paradigma que se ponen en marcha a día de hoy tienden a formar parte de la educación formal. El Aprendizaje a lo largo de la Vida ha de "garantizar el futuro económico (...), contribuir a la civilización de la sociedad y desarrollar la dimensión 
espiritual de la vida, y promover la ciudadanía activa" (DfEE, 1998:7). García Ruíz (2005) señala como uno de los atributos más valiosos e intrínsecos de la naturaleza del Aprendizaje a lo largo de la Vida la democratización del saber a todos, lo cual supone que los adultos sean considerados el colectivo prioritario de atención para las medidas políticas encargadas de su implantación. No obstante, y debido en parte a la vulnerabilidad conceptual de este paradigma, sigue habiendo autores que consideran que la Educación de Adultos conforma "un ámbito débil y generalmente menospreciado de la escena educativa oficial" (Giere y Piet, 1997:3-4, citado por Field, 2000:22 y García Ruíz, 2005).

Etcheverría Arroyo (2007) sustenta que el concepto de Educación Permanente se encuentra dividido entre aquellos que lo entienden como Educación a lo largo de la Vida y los que lo conciben como Aprendizaje a lo largo de la Vida. Asimismo, siguiendo las aportaciones de este autor, en el primer caso, se denota una influencia directa del Informe de la Comisión Internacional de Educación para el Siglo XXI (Informe Delors) en el que se expresa un continuum educativo descrito como:

[...] las funciones asignadas a la educación y las múltiples formas que puede tomar, englobando, desde la infancia al fin de la vida, todas las ofertas que permiten a cada persona acceder a un conocimiento dinámico del mundo, de los demás y de sí mismo" interrelacionando los considerados cuatro "... pilares fundamentales del aprendizaje: aprender a conocer, a hacer, a vivir y a convivir con los demás y aprender a ser. (UNESCO, 1995)

En el segundo caso, por otro lado, es perceptible la relevancia que le fue conferida por la OCDE al concepto de Aprendizaje a lo largo de toda la Vida. El aprendizaje abarca el desarrollo personal y social en todas sus formas y contextos.

Consiste en una aproximación sistémica que acompaña los niveles de conocimientos y de competencias a adquirir, independientemente de la edad de los sujetos. Su énfasis radica en la necesidad de motivar a la infancia para instruirse durante toda la vida y orientar los esfuerzos de manera que todos los adultos tengan la oportunidad de hacerlo. (OCDE, 1996)

El Aprendizaje a lo largo de la Vida tiene su origen a finales de la Primera Guerra Mundial, incrementándose su influencia durante los años 20 principalmente en la promoción política del concepto, el debate activo sobre la extensión de los derechos humanos a las mujeres y a los colectivos pertenecientes a la clase obrera (Field, 2000, en García Ruíz, 2005). Aunque no fue hasta los años 70 cuando toma verdaderamente parte de la política educativa. 
A consecuencia de un debate originado por la UNESCO, tuvo lugar la publicación del Informe Faure Aprender a Ser (1972), el cual supuso un hito en lo que al establecimiento y declaración oficial de los principios de la Educación a lo largo de la Vida se refiere. Para este organismo internacional, la educación debía tener lugar en toda la vida de todos los individuos y no únicamente durante un periodo de tiempo determinado y para colectivos minoritarios privilegiados. Por su parte, la OCDE prefirió aludir a la educación recurrente, consistente en la propuesta de medidas políticas enfocadas a la preparación de jóvenes para el mundo laboral, entendiendo al individuo como capital humano. Esto surge como consecuencia de las elevadas tasas de desempleo, persiguiendo la finalidad de atenuar la situación provocada por la crisis económica.

García Ruíz (2005), por su parte, mantiene que aquellos ideales de carácter humanista que fueron inspiradores de la Educación a lo largo de la Vida referida en el Informe Faure, se sustituyeron por una serie de modificaciones que perseguían la promoción de una fuerza laboral enfocada a la productividad, la eficiencia para acrecentar la competitividad económica nacional y el nivel de vida. Tanto la perspectiva de potenciación de la formación de la ciudadanía como la del logro de la fuerza laboral competente y competitiva contribuyen actualmente a las políticas actuales. Asimismo, el paradigma del Aprendizaje a lo largo de la Vida se encuentra estrechamente relacionado con dos de las funciones sociales primordiales de la educación, el cambio y el control social.

Field (2000:134) recoge en su trabajo cuatro estrategias esenciales a las que se habrá de atender en el caso de implantar el Aprendizaje a lo largo de la Vida en los sistemas educativos, las cuales se muestran a continuación:

Reformulación del rol de la escolaridad en la sociedad del aprendizaje.

$\checkmark$ Incremento de la participación en el aprendizaje adulto.

$\checkmark$ Construcción de una ciudadanía activa invirtiendo en capital social.

$\checkmark$ Consecución de la búsqueda de significado.

\subsection{Perspectiva y evolución histórica en Alemania}

En los inicios del siglo XIX Alemania posee un fuerte foco de influencia prusiana de carácter educativo que inevitablemente irradia en el resto de países germanófonos centroeuropeos. Concretamente en el año 1811 tiene lugar en Prusia la paradigmática Reforma de Humbolt, lo cual supone la supresión de las escuelas elementales del momento, estableciéndose así un sistema escolar graduado y único para todos los 
ciudadanos (Gómez R. de Castro, 1994). La escuela cumple eficientemente con la función alfabetizadora, y es a partir del siglo XX cuando toma una vía más enfocada a la extensión cultural y la ayuda para la inserción laboral. En Alemania, antes de la II Guerra Mundial, es posible distinguir tres fases en el ámbito de la Educación de Adultos.

En la primera fase, la Revolución Industrial resulta amenazante para artesanos y campesinos, provocando a su vez el surgimiento de asociaciones destinadas a la conservación de la producción de los mismos. No obstante, esta situación va sufriendo cambios progresivamente, cuya causa es principalmente el incremento del proletariado industrial aproximadamente a mediados del siglo XIX. Durante esta época, las políticas formativas abarcan, por un lado, la educación elemental y, por otro, la formación profesional como vía para superar dificultades económicas y laborales. Asimismo también cobra importancia la formación política con la finalidad de implantar un orden social democrático en las estructuras feudales del momento (Mollenhaver, 1973 en Gómez R. de Castro, 1994). A raíz de la Revolución de marzo de 1848 se finaliza esta etapa para dar comienzo a otra nueva.

La segunda fase comprende los periodos de la Restauración y el Imperio Alemán, llegando a su fin en 1918. Tras la Revolución de 1848 se produce un notable incremento de las diferencias entre burgueses y proletarios, no obstante, en lo referente a la organización de la formación de adultos se conservan actitudes colaborativas para lograr la constitución democrática del Estado, en contra de la Restauración. En 1869 terminan por disolverse los congresos comunes, lo cual supone la separación, tanto en contenidos como en líneas de organización, entre las concepciones de Educación Popular de los liberal-burgueses y de las de los socialistas y socialdemócratas, respectivamente. En 1871 se funda la Sociedad para la extensión de la Educación Popular, que consiste en una reacción ante los "cada vez más agudos perfiles de las clases sociales" (Gómez R. de Castro, 1994:182), siendo esto la causa de que la educación popular burguesa deje a un lado los principios antifeudalistas, anticlericales y emancipatorios. De forma paralela a la formación burguesa, se desarrolla una modalidad de Educación de Adultos enfocada al colectivo de los obreros, entendida como formación facilitadora de la inserción social y que haga aumentar la influencia para el camino hacia una sociedad de corte socialista. La Formación de funcionarios dentro de los sindicatos, sancionada por la ley de 1920, constituye un ejemplo de la misma (Feidel-Mertz, 1972 en Gómez R. de Castro, 1994).

Finalmente, la tercera fase se corresponde con el periodo de la República de Weimar. La Sociedad para la extensión de la educación popular a partir de 1918 acentúa la formación individual y humanista. En las escuelas superiores populares cada vez son más las personas que 
asisten como oyentes ocasionales de conferencias, además de asociaciones de trabajadores. Se perseguía el perfeccionamiento y el desarrollo de las cualidades personales del individuo. Es en 1919 cuando la Constitución de Weimar potencia la creación de la Volkhochschule desde el Gobierno Federal, los Länder y ayuntamientos.

La Volkhochschule está muy extendida por todo el país y sigue siendo a día de hoy la institución dedicada a la Educación de Adultos más representativa de Alemania. El objetivo que persiguen es "la adquisición de conocimientos y la profundización en las experiencias vividas, la transmisión de información contrastada, la potenciación del pensamiento propio e independiente de las personas y el ejercicio de las capacidades creativas" (Gómez R. de Castro, 1994:183).

En la República Federal Alemana, es posible distinguir dos periodos dentro de la Educación de Adultos (Gómez R. de Castro, 1994). En primer lugar, hasta finales de la década de los 50 del siglo XX, las nuevas iniciativas, concepciones e intentos por retomar el rumbo de la formación de las personas adultas que ya existía en la República de Weimar brillan por su ausencia. Todo ello se avivaba con la carencia de fundamentos legales y ayudas presupuestarias, por lo que la Educación de Adultos pasa a contemplarse como un lujo superfluo cuyo patrocinio no ha de ser necesariamente proporcionado por el sistema de formación general de la República Federal Alemana. No fue hasta la segunda etapa, justo después de la II Guerra Mundial, cuando como consecuencia de las problemáticas económicas, de la Segunda Revolución Industrial y de la falta de mano de obra cualificada se volcaron esfuerzos en dirección a la formación profesional industrial, pasando así la formación política a un segundo plano.

\section{EDUCACIÓN SUPERIOR}

Los estudiantes adultos representan una categoría dentro de aquellos de Educación Superior que es complicado definir pues, a nivel europeo, no existe una definición para dicho término que sea comúnmente aceptada e identifique tal categoría en términos de edad. De hecho, la mayoría de los estudiantes que inician estudios superiores suelen haber alcanzado ya la mayoría de edad legalmente establecida. Sin embargo, se consideran estudiantes adultos a aquellos que son mayores que el típico estudiante de Educación Superior y que ha vuelto a formar parte de la misma tras un periodo de tiempo fuera del sistema.

El Aprendizaje a lo largo de la Vida, tal y como se recoge en EURYDICE (2011), ha conformado una parte integral de las pretensiones del proceso de Bolonia y su importancia ha sido subrayada en todas las communiqués que fueron sucediendo la Declaración de Bolonia de 
1999. Más recientemente ha obtenido un lugar especial Lovaina/Louvainla-Neuve Communiqué (2009) lo cual resaltó la importancia de la implementación de las políticas de aprendizaje a lo largo de la vida.

It is always a good time to learn (European Commission, 2007) consiste en un plan de acción para la Educación de Adultos que incluye referencias sobre la participación de adultos en la Educación Superior y demanda medidas para ampliar las oportunidades de estos estudiantes que quieran embarcarse en estudios superiores.

La Educación Superior se concibe a menudo como el sector más desafiante cuando se trata de implementar trámites para el reconocimiento de aprendizajes previos no formales y/o informales. Este proceso requiere que el reconocimiento de cursos no-formales, experiencia laboral y experiencias vitales pueden ser equivalentes al aprendizaje producto de años de educación formal, y puede cualificar a estudiantes para ingresar en la Educación Superior para progresar en su formación permanente.

En lo referente a las infraestructuras legislativas relacionadas con el reconocimiento y la convalidación del aprendizaje previo no formal y/o informal adquirido en Europa en el ámbito de la Educación Superior se aborda desde varias vías: la legislación puede ser directiva, requiriéndole explícitamente a las instituciones adoptar el proceso en cuestión; puede ser permisiva, dejando que las instituciones tomen medidas de precaución si así lo desean; y puede no hacer referencia a nada (Eurydice, 2011). El reconocimiento de aprendizaje previo en el ámbito de la Educación Superior puede tener dos propósitos diferentes: En primer lugar, permitirle a los estudiantes ser admitidos en una institución de Educación Superior y, en segundo lugar, permitirles demostrar que reúnen los requisitos, total o parcialmente, de un programa de Educación Superior.

En el mapa 1 se puede observar un mapa sobre aquellos países europeos en los que existen vías alternativas ofertadas a los estudiantes no tradicionales para la Educación Superior, basada en datos de 2009/2010. El que existan vías alternativas implica que existe, por lo menos, una vía para acceder a la Educación Superior. El que no existan vías alternativas significa que se precisa un certificado de Educación Secundaria para acceder Estudios Superiores. En el mapa 2 se aprecia de una forma más clara y visual la afirmación acerca de las infraestructuras legislativas a las que en líneas previas se ha hecho alusión. 
Mapa 1. Vías alternativas para la Educación Superior a candidatos no tradicionales, 2009/2010. Fuente: Eurydice, 2011:45

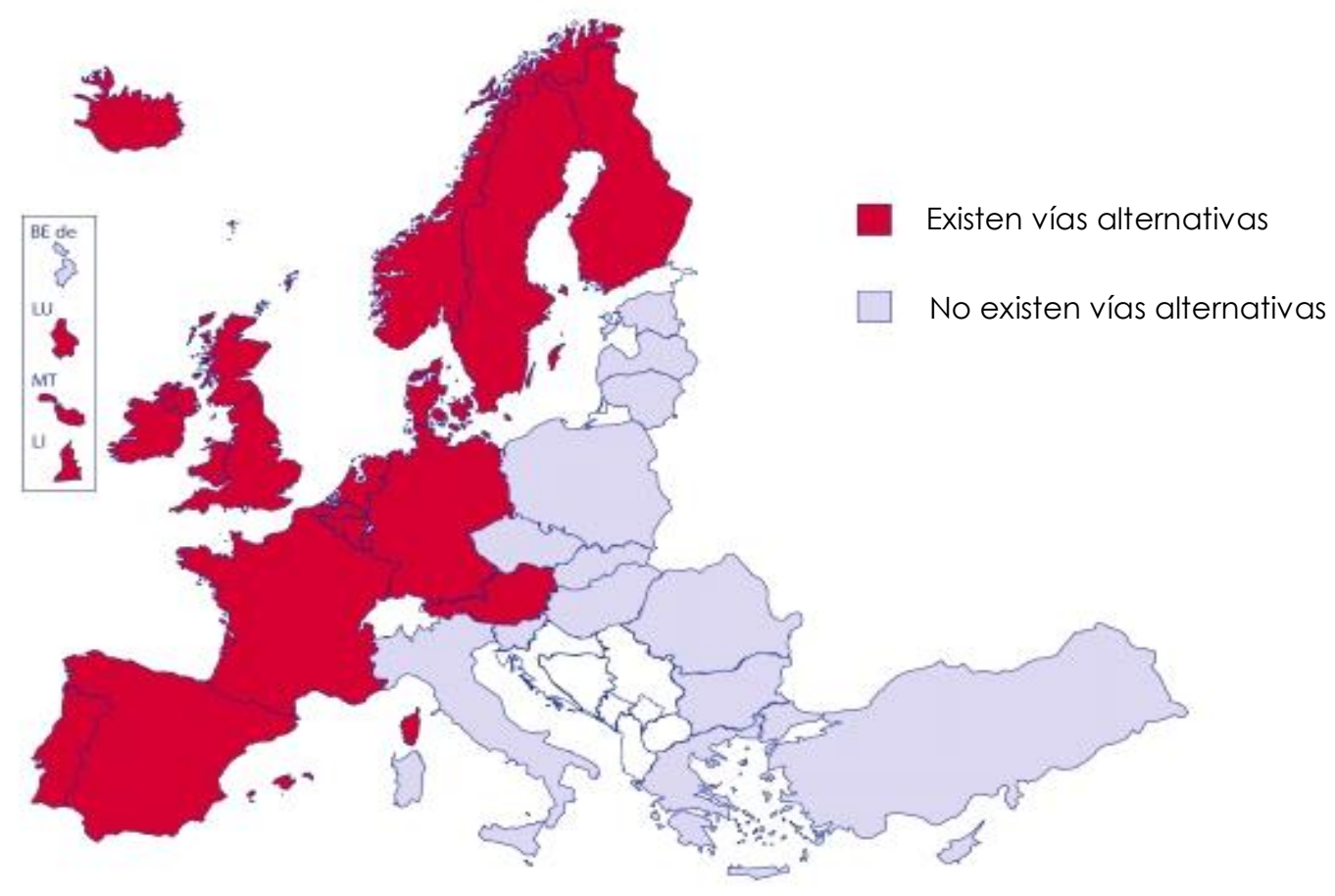

Mapa 2. Infraestructuras legislativas para la validación de aprendizajes previos no formales e informales en la Educación Superior, 2009/2010. Fuente: Eurydice, 2011:47

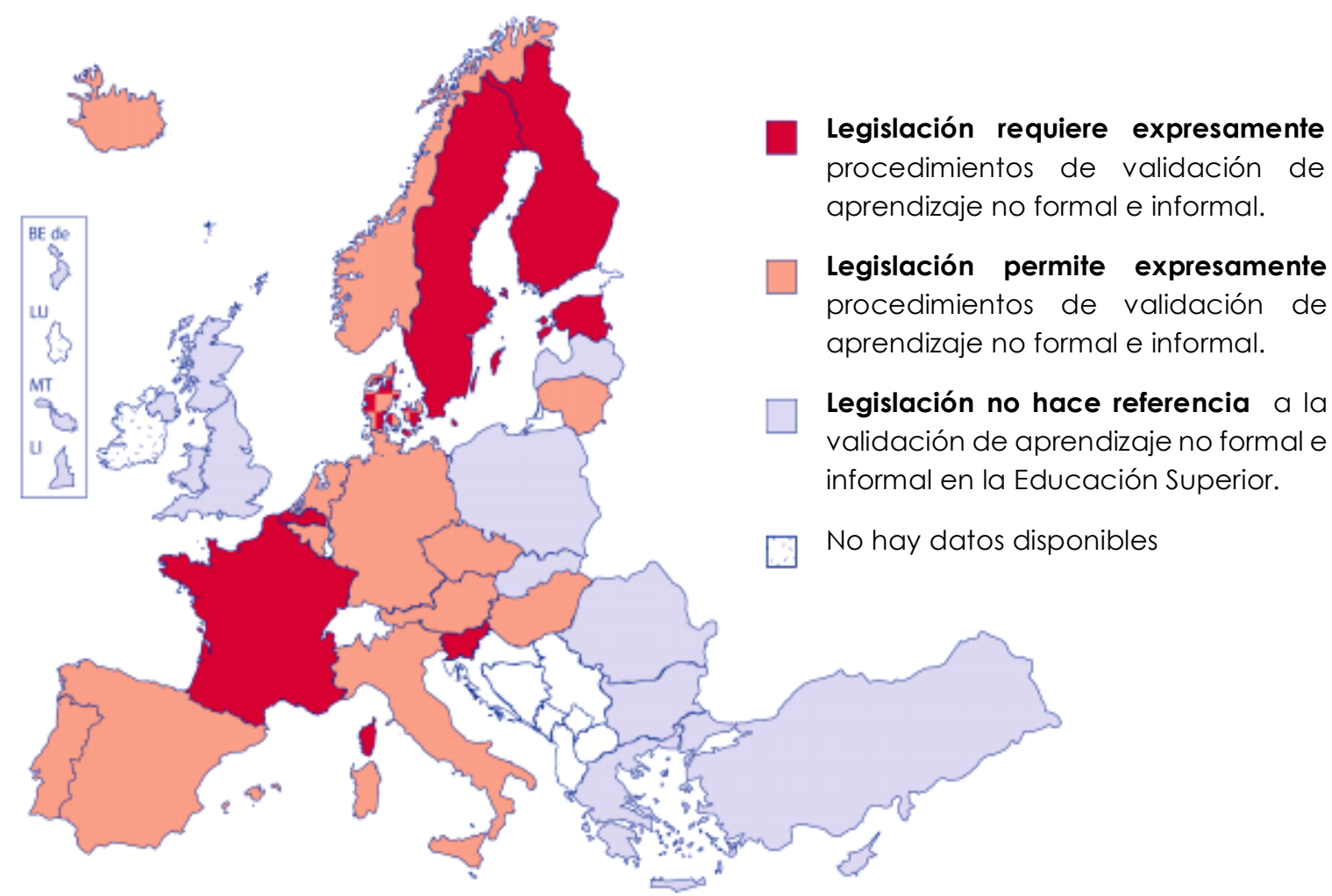




\subsection{Educación Superior de Adultos en el ámbito universitario en Alemania}

Desde 1980 está creciendo un interés político y académico por un grupo de estudiantes concebido como 'no tradicionales', pero siempre ha sido difícil definir de manera precisa qué se entiende por estudiante no tradicional, especialmente desde una perspectiva comparativa internacional. Para entender la noción particular alemana de los estudiantes no tradicionales es útil hacer distinción entre cinco aspectos diferentes (Kasworm 1993, en Wolter 2012).

En primer lugar, la edad constituye una aproximación simple pero extendida. Define a este tipo de estudiantes en la línea de una edad cronológica, normalmente extrapolada a aquellos mayores de 25 años en el momento de matriculación. En este contexto, abrir el acceso a la Educación Superior supone una mezcla de edades en la composición del cuerpo estudiantil.

Por otro lado está la participación, que de acuerdo con la misma, el alumnado no tradicional lo constituyen grupos infrarrepresentados en relación con su participación en la Educación Superior (con trasfondos migratorios, con diversidad funcional, etc.). Una política abierta de acceso implica una estrategia compensatoria para lograr la inclusión social y reducir la desigualdad en los patrones y las tasas de participación.

El tercer lugar lo ocupa el ciclo vital, concepto referente a los escenarios de los caminos hacia la Educación Superior, abarcando a menudo no sólo la trayectoria escolar, sino periodos de prácticas y experiencia laboral, etc. Desde esta perspectiva, el objetivo de la política sería promover la diversidad experimental en el alumnado.

En cuanto al acceso y la admisión, cabe afirmar que normalmente los estudiantes no tradicionales acceden a la universidad en un momento posterior del tiempo que les correspondería, tras un procedimiento especial de admisión o por convalidación de experiencia laboral previa. En este caso, la apertura de acceso significaría un incremento de la permeabilidad entre la Formación Profesional y la Educación Superior.

Finalmente, de acuerdo con la aproximación a las modalidades de estudio, los estudiantes no tradicionales son, principalmente, aquellos que estudian en ámbitos no formales así como a tiempo partido, a distancia o de forma paralela a su trabajo. Para promover a los estudiantes no tradicionales desde esta perspectiva habría que organizar cursos y estudios de una forma más flexible y orientada a la demanda. 
De acuerdo con las aproximaciones anteriormente mencionadas, pueden distinguirse seis diferentes grupos de participantes con los que se cubriría la noción de estudiante no tradicional alemán (Wolter, 2012):

$\checkmark$ La segunda vía educativa: Esta categoría recibe a aquellos estudiantes que dejaron la escuela antes del Abitur pero que posteriormente completaron la Formación Profesional y asistieron al Abendgymnasium o Kolleg, una de las posibilidades de Educación Secundaria para adultos, tras un periodo de experiencia laboral. Esta vía se encuentra regulada por la legislación escolar. En el estudio de la OCDE (1987) se les denomina second chancers 5 .

$\checkmark$ Tercera vía educativa: En alemán Dritter Bildungsweg se refiere a aquellos estudiantes que, sin tener el Abitur, son admitidos por uno de los procedimientos especiales de admisión en el nivel universitario. También son considerados second chancers aunque a diferencia del grupo anterior, este es materia de la legislación propia de la Educación Superior y supone ser el núcleo de los considerados, bajo juicio alemán, estudiantes no tradicionales.

$\checkmark$ Egresado escolar doblemente cualificado: Es otra agrupación híbrida que se refiere a estudiantes que han obtenido el Abitur y que dirigen sus estudios hacia la Formación Profesional antes de trasladarse a la Educación Superior. El objetivo principal de estos estudiantes es cultivar su futuro laboral mediante competencias profesionales y una titulación. La terminología empleada en el estudio de la OCDE (1987) los denomina deferrers 6 .

$\checkmark$ Disposiciones de estudio no tradicionales: Con independencia de la cualificación formal y titulación, los estudiantes pueden optar por diversas modalidades de estudios, entre las cuales la enseñanza a distancia y a tiempo parcial conforman un ejemplo. En Alemania únicamente una pequeña cantidad de provisiones tan flexibles están organizadas formalmente, siendo considerable el número de estudiantes a tiempo parcial. Por ello, si la definición se refiriera a la modalidad de estudio el número de estudiantes no tradicionales sería sobreestimado.

$\checkmark$ Participantes en Formación Superior Continua: Otra categoría es aquella en la que se incluyen como estudiantes no tradicionales a los que toman parte en la formación continua o permanente. La mayoría de este colectivo ya posee una primera titulación y vuelven a ingresar en la Educación superior con diversos

\footnotetext{
5 Concepto empleado por la OCDE (1987) para denominar a aquellas personas cuyo ingreso en la Educación Superior es posterior, haciendo referencia a la "segunda oportunidad" que para ello les ha sido prestada.

6 Concepto adoptado por la OCDE (1987) para denominar a los estudiantes que acceden a la Educación Superior a posteriori, refiriéndose a la "prorrogación" de los estudios que en la misma tienen lugar.
} 
propósitos. La OCDE (1987) se refiere a ellos como returners. Este tipo de formación se institucionaliza en tres niveles: en primer lugar, como un curso que conduzca a una titulación (normalmente máster), cursos no vinculados a una titulación (tienen su propio certificado) o como prestaciones públicas de orientación general (en inglés PUSH provisions ${ }^{7}$ ).

$\checkmark$ Estudiantes mayores: A este grupo pertenecen los estudiantes que se matriculan posteriormente a su jubilación. Pueden inscribirse en cursos habituales o en programas específicos, a veces en instituciones especiales como las Universidades de la Tercera Edad. Normalmente no suelen aspirar a la obtención de una cualificación profesional formal, incluso si su propósito es la obtención de una titulación formal; Wolter (2007b) manifiesta que suelen estudiar por interés y realización personal.

En todos los Länder alemanes se ha iniciado un procedimiento especial de admisión, en la mayoría de los cuales el nivel es mínimo. No obstante, los métodos particulares para estas vías de acceso varían según los Estados Federados o Länder, aunque tomando como referencia el trabajo de Nickel y Leusing (2009), se puede comprobar como las vías de acceso a la Educación Superior sin necesidad de haber realizado el Abitur convergen en cuatro modalidades, a saber:

El acceso directo a la Universidad para personas especialmente cualificadas profesionalmente (Direkter Hochschulzugang für beruflich besonders Qualifizierte): Actualmente el acceso a la Universidad por esta vía es posible en 14 Länder teniendo aprobado un examen de maestría - título equivalente. La apertura del acceso a la Universidad, especialmente para estos destinatarios, ha estado fuertemente impulsado en los últimos años.

El acceso a la Universidad mediante un examen de ingreso (Hochschulzugang über eine Zulassungsprüfung): A excepción de Berlín, el resto de los Länder ofrecen esta posibilidad como acceso a la Universidad. Las condiciones de admisión para el examen de ingreso abarcan desde un determinado título educativo (Hauptschule, Mittlere Reife) hasta un examen de maestría o similar. Las infraestructuras para los exámenes se encuentran regladas por la legislación de Educación Superior del Land (Landeshochschulgesetz). En los Länder se identifican, asimismo dos variantes:

El examen de ingreso como única vía de acceso a la Universidad para personas sin Abitur y sin Fachhochschulreife 8 .

\footnotetext{
7 PUSH Provisions promueven el Public Understanding of Sciences and Humanities (Entendimiento público de ciencias y humanidades).

8 "Las escuelas superiores especializadas suelen conformarse con una titulación que ellas mismas expiden tras la aprobación de pruebas organizadas por y en ellas: es el llamado 
$\checkmark$ Examen de ingreso como alternativa al estudio de prueba (Probestudium).

El acceso a la Universidad mediante un estudio de prueba (Hochschulzugang über ein Probestudium): Esta modalidad destinada principalmente a estudiantes cualificados profesionalmente es la prioritaria desde la década de los 90 en los Länder del norte de Alemania, así como en Berlín, y actualmente existe en 6 de ellos. Según este método, una vez realizado el examen de inscripción, se procede a la inscripción de los candidatos durante un periodo de uno a cuatro semestres en la universidad en cuestión. Una vez finalizado el estudio de prueba se lleva a cabo una evaluación de conocimientos y la matriculación definitiva. Al igual que en la modalidad anterior, se pueden identificar dos variantes:

$\checkmark$ Estudio de prueba como única vía de acceso a la Universidad para personas sin Abitur y sin Fachhochschulreife.

$\checkmark$ Estudio de prueba como alternativa al examen de ingreso.

El acceso a la Universidad mediante un test de aptitudes académicas (Hochschulzugang über die Begabtenprüfung): Esta prueba es una oportunidad para adquirir el Allgemeine Hochschulreife sin necesidad de asistir a un centro de bachillerato nocturno (Abendgymnasium) o instituciones educativas similares para alcanzar el Abitur, sino aprobando un examen individual.

Sin embargo, en el trabajo de Nickel y Leusing (2012) se recogen las modificaciones establecidas por el KMK 2009 (Kultusministerkonferenz) referentes al acceso a la Universidad para aquellos que no poseen el Abitur, las cuales pueden resumirse en las siguientes:

$\checkmark$ Los poseedores de un título de capacitación laboral superior, maestría o título similar tienen derecho a acceder a la Universidad.

$\checkmark$ Otros estudiantes interesados cualificados laboralmente con mínimo dos años de Formación Profesional y como mínimo tres años de experiencia laboral pueden obtener el derecho a acceder a la Universidad relacionada con su rama, tras el cual obtiene un juicio declarativo de aptitudes (Eignungsfeststellungsverfahren).

$\checkmark$ Los Länder pueden ampliar las reglas de acceso a la Universidad, sobre todo para el reconocimiento de títulos de formación avanzada que otorguen el derecho a entrar en la Universidad.

A modo de resumen $y$, para que no resulte tan complejo, se muestra a continuación una tabla en la que se representa la situación del

'certificado de madurez superior especializada' o Fachhochschulreife". García Garrido (2005:96).

Revista Fuentes, 17, Diciembre 2015; pp. 89-112 
acceso a la Universidad antes y después de las reformas de 2009 establecidas por el KMK:

Tabla 1. Situación antes y después de la reforma del KMK

\begin{tabular}{|c|c|}
\hline Antes de la reforma del KMK (2009) & Tres años tras la reforma del KMK \\
\hline $\begin{array}{l}\text {-Acceso a la Educación Superior para } \\
\text { expertos y cualificados profesionales } \\
\text { en la materia en } 14 \text { Bundesländer. } \\
\text { Normativa sobre la edad mínima y el } \\
\text { domicilio principal (Cláusulas de } \\
\text { menores propias del Land o } \\
\text { Landeskinderklauseln). } \\
\text { Seis Bundesländer ofrecen la } \\
\text { posibilidad de realizar estudio de } \\
\text { prueba. } \\
\text { No existe reconocimiento mutuo de } \\
\text { admisión y acceso a instituciones } \\
\text { superiores sin el Abitur en otros } \\
\text { Bundesländer. } \\
\text { Siete Bundesländer posibilitan el } \\
\text { reconocimiento de cuidados y } \\
\text { educación. }\end{array}$ & $\begin{array}{l}\text { A personas con estudios de } \\
\text { formación profesional finalizados y } \\
\text { con prácticas profesionales de varios } \\
\text { años se le concede el derecho de } \\
\text { acceso a la Educación Superior en los } \\
16 \text { Bundesländer. } \\
\text { Los expertos y cualificados } \\
\text { profesionales tienen derecho al } \\
\text { acceso a instituciones de Educación } \\
\text { Superior en } 14 \text { Bundesländer. } \\
\text { Siete Bundesländer ofrecen la } \\
\text { posibilidad de realizar estudio de } \\
\text { prueba. } \\
\text { Reconocimiento mutuo de admisión } \\
\text { y acceso a instituciones superiores sin } \\
\text { el Abitur en } 11 \text { Bundesländer. } \\
\text { Diez Bundesländer posibilitan el } \\
\text { reconocimiento de cuidados y } \\
\text { educación. }\end{array}$ \\
\hline
\end{tabular}

\section{ACCESO A LA UNIVERSIDAD EN LA WESTFÄLISCHE WILHELMS-UNIVERSITÄT MÜNSTER.}

A continuación se va a proceder a examinar las diferentes posibilidades de Acceso a la Universidad para aquellos estudiantes adultos que no hayan realizado el Abitur y deseen ingresar en una institución de enseñanza superior de esta índole.

La Westfälische Wilhelms-Universität (WWU) es una de las cinco mayores universidades de Alemania (Münster in Westfalen, 2014), que acoge cada curso académico a más de 36.000 estudiantes alemanes y extranjeros (Statistisches Bundesamt, 2013; WWU, 2014) de carácter público cuya fecha de fundación se sitúa en el año 1780. Esta institución se encuentra localizada en la ciudad de Münster, la mayor del territorio de Münsterland (Encyclopaedia Britannica, 2014) y perteneciente al Land de Renania del Norte y Westfalia (Nordrhein-Westfalen) situado al noroeste de Alemania.

En la WWU es posible distinguir tres modalidades de acceso a la misma: Expertos (Meister) o cualificados similares; solicitantes 'fieles' a la titulación (Factreve); y solicitantes 'no fieles' a la titulación (nicht Fachtreve). 


\subsection{Expertos (Meister) o cualificados similares}

Esta modalidad de acceso se establece de acuerdo con el $\S 2$ del denominado Berufsbildungshochschulzugangsverordnung o, en Español, Reglamento de Acceso a la Educación y Formación Superior.

Los individuos pertenecientes a este grupo tienen autorizado el acceso a todas las carreras, posibilitándoles el acceso directo a los estudios superiores sin necesidad de realizar un examen previo. No obstante, dentro de esta categoría es posible encontrar otros tres subgrupos:

$\checkmark$ Para acceder a las titulaciones con plazas limitadas a nivel nacional (medicina, farmacia, odontología), es recomendable registrarse en la Fundación para el Acceso a la Educación Superior (Stiftung für Hochschulzulassung). Es posible participar en el proceso de adjudicación con una calificación superior o igual a 49 . Sin embargo también existe la posibilidad, mediante la realización de un examen de acceso, de mejorar la calificación obtenida y, por consiguiente, las oportunidades. La solicitud para el examen de acceso para el semestre de verano (Sommersemester) ha de ser enviada antes del 1 de octubre, y antes del 1 de abril para el semestre de invierno (Wintersemester).

$\checkmark$ Para acceder a las titulaciones con plazas limitadas a nivel local, es necesario presentar una solicitud a través de la página web de la universidad ${ }^{10}$ dentro del plazo establecido. Así, para el semestre de invierno el plazo se iniciaría a principios de mayo, finalizando el 15 de julio; para el semestre de verano se iniciaría a principios de noviembre, hasta su vencimiento el 15 de enero. Normalmente suele ser más elevado el número de aspirantes que el de plazas ofertadas, caso en el que una comisión interna departamental se encarga de la toma de decisiones sobre su aprobación.

$\checkmark$ Para acceder a las titulaciones de libre acceso, es posible matricularse para la realización de estudios de prueba (Probestudium) desde mediados del mes de junio hasta principios de octubre -para comenzar en el semestre de inviernoy desde mediados de enero hasta finales de marzo -para el semestre de verano. Es posible cumplimentar la inscripción tanto de forma presencial como a través de internet ${ }^{11}$.

\footnotetext{
9 Las calificaciones en Alemania se puntúan del 1 al 6 (Berengueras Pont, 2011), correspondiéndose en este caso la puntuación de 4 con el mínimo para participar en el proceso al que nos referimos.

10 http://www.uni-muenster.de/studium/bewerbung/index.html

11 http://www.uni-muenster.de/studium/einschreibung/index.html
}

Revista Fuentes, 17, Diciembre 2015; pp. 89-112 


\subsection{Solicitantes 'fieles' a la titulación (Factreve)}

La presente modalidad de acceso fue establecida de acuerdo con el $\S 3$ del Berufsbildungshochschulzugangsverordnung.

Esta opción permite el ingreso directo a los estudios de Enseñanza Universitaria a los solicitantes que dispongan de una titulación de Formación Profesional y cuyas habilidades les permitan llevar a cabo actividades técnicas y profesionales. A estos colectivos se les atribuye en alemán la denominación Fachtreven, concepto que hace referencia a la fidelidad profesional de los solicitantes para con el área de trabajo o, en este caso, estudios que solicita. Entre los individuos que forman parte de este colectivo se incluyen aquellos cuyos estudios de Formación Profesional (Berufsausbildung) han llegado a su fin, aquellos que hace como mínimo dos años que finalizaron este tipo de estudios y que, a posteriori, hayan desempeñado durante tres años un puesto técnico profesional dentro de la misma área de trabajo. Al igual que en la categoría descrita anteriormente, en esta se engloban otros dos subgrupos, muy similares a los anteriores:

$\checkmark$ Para obtener el acceso a aquellas titulaciones cuyo número de plazas a nivel nacional están limitadas (medicina, farmacia, odontología), se recomienda inscribirse en la stiftung für Hochschulzulassung. Es posible participar en el proceso de adjudicación de plaza siempre y cuando la calificación numérica sea igual o superior a 4. No obstante, existe asimismo la posibilidad, de mejorar la calificación obtenida y, por consiguiente, las oportunidades a través de la realización de un examen de acceso. La inscripción para el examen de acceso ha de ser enviada directamente a la facultad de interés. El periodo indicado para ello finaliza el 1 de octubre para el Sommersemester, y el 1 de abril para el Wintersemester.

$\checkmark$ Para acceder a aquellas titulaciones cuyo número de plazas a nivel local están limitadas, se requiere la presentación de una solicitud a través de la dirección web anteriormente indicada en el plazo para ello establecido -desde inicios de mayo hasta el 15 de julio para el semestre de invierno y de principios de noviembre hasta el 15 de enero para el semestre de verano. Es habitual que el número de aspirantes sea superior al de las plazas ofertadas. En este caso una comisión interna departamental pasa a ocuparse de las decisiones pertinentes para su aprobación.

\subsection{Solicitantes 'no fieles' a la titulación (nicht Fachtreve)}

Esta última modalidad de acceso se fundamenta de acuerdo con el $\S 4$ del Berufsbildungshochschulzugangsverordnung mencionado en las otras dos formas anteriormente descritas. 
Mediante esta modalidad se permite que aquellos solicitantes que se encuentren en posesión de un título de Formación Profesional de, como mínimo, dos años de duración y tres años de experiencia profesional (que no tiene que estar estrictamente relacionada con la profesión estudiada) accedan a estudios universitarios en especialidades que no se correspondan con la vía profesional que hasta el momento han tomado. Para situaciones de empleo realizadas, como mínimo, a tiempo parcial, las habilidades profesionales serán proporcionalmente acreditadas. Como cualidad profesional se reconocen situaciones en las que el individuo cuente con independencia propia y sea el responsable principal de un hogar familiar en el que se encuentre, al menos, un menor de edad o pariente con diversidad funcional que precise ayuda y cuidados específicos, debido a una situación de dependencia. En esta categoría es posible hacer distinción de otros tres subgrupos, al igual que en las modalidades anteriores, a saber:

$\checkmark$ Para ingresar en titulaciones con plazas limitadas a nivel nacional (medicina, farmacia, odontología), se recomienda registrarse en la Stiftung für Hochschulzulassung. No obstante, es necesario realizar un examen de acceso previo a través del cual se obtendrá una nota que le permitirá a los solicitantes poder participar en el proceso de adjudicación de plazas.

$\checkmark$ Para acceder a las titulaciones con un limitado número de plazas disponibles, se requiere la realización de una prueba de acceso.

$\checkmark$ Para lograr la admisión a las titulaciones de libre acceso, se ofrece la posibilidad de elegir entre la realización de una prueba de acceso $u$ optar por un periodo de prueba. Una vez finalizado con éxito el periodo de prueba, la inscripción en la titulación deseada será definitiva.

\section{CONCLUSIONES}

A modo de colofón cabe hacer resaltar el carácter reciente de los conceptos objeto de estudio, pues son acuñados en Europa en torno al Siglo XIX. En relación con esto, resulta conveniente aludir al desacuerdo existente entre los países a la hora de definirlos. Esto es debido a discrepancias entre factores como la edad, el nivel educativo, la formalidad del aprendizaje, etc. que se encuentran en estrecha relación con los términos, pues son determinantes del mismo. Ello nos lleva a que, actualmente, debido a la evolución histórica de los términos y al desarrollo de la sociedad, las diferencias se hagan cada vez más notorias, dirigiéndose el foco de interés hacia múltiples vertientes como pueden ser: el Aprendizaje a lo largo de la Vida, derivando en ocasiones hacia la Educación Permanente o, la Educación Superior de Adultos en el Ámbito Universitario, abriéndose así nuevas puertas mediante la creación y la posterior mejora de las vías de Acceso a la Universidad. 
Entre las fortalezas más destacadas del sistema educativo alemán para con la temática concerniente, resulta sustancial tener presente algunos estudios de Eurydice, entre otros, en los que se aprecia que en Alemania, al igual que en otros países de la zona norte y oeste del continente europeo, los estudiantes considerados "no tradicionales" cuentan con mayores posibilidades de acceso a enseñanzas de niveles educativos superiores que en otros países de Europa, concretamente aquellos geográficamente localizados en el sur y el este.

Asimismo, en cuanto a las infraestructuras legislativas para la validación de aprendizajes previos no formales e informales en la Educación Superior, aunque las diferencias entre países se antojan más heterogéneas, la situación de Alemania se asemeja a la de otros países europeos con sistemas educativos muy disímiles entre sí como España, Portugal, Italia, Islandia, Noruega o Austria, entre otros. Debiéndose este fenómeno a la que la legislación vigente permite llevar a cabo procedimientos de validación de aprendizajes de carácter no formales e informales, en lugar de convertirlos en requisitos que deban cumplirse expresamente, como sucede en Francia, Suecia, Finlandia, Estonia, Eslovenia y algunas zonas de Dinamarca.

Por otro lado, cabe subrayar la existencia de diferencias y similitudes encontradas en las vías de Acceso a la Universidad para estudiantes adultos en la WWU, siendo unas más notorias que otras. Una de las más significativas sería el nivel educativo de los solicitantes, pues constituye el factor principal de clasificación de los mismos para acceder a las titulaciones deseadas. Consideramos pues este fenómeno un elemento crucial a la hora de valorar la admisión de nuevos estudiantes en diferentes titulaciones académicas, estableciendo diferencias en función no únicamente de los méritos personales, sino también de las exigencias propias de la titulación deseada. En otros países como es el caso de España, el Acceso a la Universidad depende más de las edades de los futuros estudiantes universitarios, en lugar de la formación previa de la que dispongan, aunque constituya también un elemento influyente.

Tampoco pasa desapercibida la subdivisión existente dentro de cada nivel especificado, atendiendo a si se desea ingresar en una titulación con plazas limitadas a nivel nacional, local o si son titulaciones de libre acceso. Las diferencias entre una y otra son significativas, variando en factores relativos al nivel de exigencia, el cual resulta más elevado en las titulaciones con plazas limitadas a nivel nacional mientras que en las titulaciones de libre acceso los requisitos a cumplir son destacadamente menos exigentes que las demás. Si volvemos la mirada hacia otros países, como el nuestro, nos daremos cuenta de que en las pruebas de acceso a la universidad para estudiantes adultos "no tradicionales", no suele influir directamente el límite de plazas existentes para las diferentes titulaciones universitarias en los periodos previos al 
examen, sino que llega a posteriori. En Alemania, sin embargo, ello supone ya un sesgo en tanto a la preparación específica de los solicitantes para acceder a un área de titulaciones en concreto.

Una vez concluido el estudio, tal vez llame la atención el hecho que, precisamente tratándose de Alemania, un país internacionalmente conocido por su prestigioso e indudablemente eficaz sistema de Formación Profesional dual, apenas se tenga en consideración el reconocimiento de competencias profesionales a partir de las experiencia previa, a modo de factor clave que facilite el acceso a los estudios superiores universitarios a aquellos estudiantes adultos "no tradicionales", aunque la valoración de las mismas sí goce, no obstante, de un balance positivo en el proceso.

Asimismo, existen estudios que ponen de manifiesto que este fenómeno lleva ya varios años en auge en algunos países, por ejemplo Francia, acercando a mayor parte de la población el aprendizaje a lo largo de la vida en los diversos niveles educativos y, especialmente, el universitario. Este hecho ha traído consigo el convertirse en un elemento innovador y muy influyente para otros países entre los que, indudablemente, también se encuentra España. Del mismo modo, teniendo en cuenta la situación de crisis en la que Europa se encuentra inmersa, esta forma de acceso podría suponer además, a juicio personal, una posible oportunidad adicional para colectivos en situación de desempleo e incluso en riesgo de exclusión laboral, sin olvidar además el elemento facilitador que brinda la gratuidad de los estudios superiores universitarios prácticamente en la totalidad de la República Federal de Alemania.

\section{REFERENCIAS BIBLIOGRÁFICAS}

Alemania. Gesetz- und Verordnungsblatt, de 8 de marzo de 2010, Verordnung über den Hochschulzugang für in der beruflichen Bildung Qualifizierte (Berufsbildungshochschulzugangsverordnung). Gesetz- und Verordnungsblatt Nordrhein-Westfalen, 12 de marzo de 2010, num. 9 pp.155-164. Recuperado de https://recht.nrw.de//mi/owa/br_vbl_detail_text?anw_nr=6\&vd_id $=12048 \&$ vd_back $=\mathrm{N}$

Berenguer Pont, M. (2011). El Sistema Educativo de Alemania. Revista de la Asociación de Inspectores de Educación de España, 15, 1-7. Recuperado de http://adide.org/revista/images/stories/PDF_15/ase15_colab01.pdf Cedefop (2008). Terminology of European education and training. A selection of 100 key terms. Luxembourg: Offi ce for Offi cial Publications of the European Communities. Recuperado de http://www.cedefop.europa.eu/en/Files/4064_EN.PDF 
Dfee (1998): Recent Thinking in Lifelong Learning.

Etcheverría Arroyo, F. D. (2007). Educación de adultos, subjetividad y transformaciones sociales: Impacto social, humano y económico de la educación de personas adultas. (Tesis inédita de doctorado). Universidad Complutense, Madrid.

Field, J. (2000). Lifelong Learning and the new Educational Order. Stoke on Trent, Trentham Books.

García Garrido, J. L. (2005). Los sistemas educativos de hoy. Madrid: Ediciones Académicas, S. A.

García Ruíz, M. J. (2005). Aprendizaje a lo largo de la vida. En García Garrido, J. L. \& García Ruíz, M. J. Temas Candentes de la Educación en el siglo XXI (pp. 43-82). Madrid: Ediciones Académicas

Gómez De Castro, F. (1994). La educación de las personas adultas a lo largo de la historia. Perspectiva nacional e internacional. En Sanz Fernández, F. (Dir.) La formación en educación de personas adultas (pp. 157-200). Madrid: UNED.

Hochschulen (2014). En Münster in Westfalen. Recuperado de http://www.muenster.de/hochschulen.html

Nickel, S, \& LEUSING, B. (2009). Studieren ohne Abitur: Entwicklungspotenziale in Bund und Ländern. Eine empirische Analyse. Arbeitspapier, 123. Recuperado de http://www.che.de/downloads/CHE_AP123_Studieren_ohne_Abitu r.p.df

Nickel, S, \& Leusing, B. (2012). Studieren ohne Abitur: Monitoring der Entwicklungen in Bund, Ländern und Hochsculen. Arbeitspaper, 157. Recuperado

de http://www.che.de/downloads/CHE_AP157_Studieren_ohne_Abitu r_2012.pdf

OECD (1996). Making Lifelong Learning a Reality for All. Paris: OECD.

Münsterland. (2014). En Encyclopaedia Britannica. Recuperado de http://www.britannica.com/EBchecked/topic/397686/Munsterland

Ranking By Country 2011-2012, Germany (2014). En Urapcenter.org. Recuperado de http://www.urapcenter.org/2011/country.php?.ccode=DE\&rank= all

Studieren Ohne Abitur (2014). En Uni-muenster.de. Recuperado de http://www.unimuenster.de/studium/bewerbung/zugangspruefung.html

Studierende An Hochschulen - Vorbericht - Fachserie 11 Reihe 4.1 Wintersemester 2013/2014 (2014). En Statistisches Bundesamt en Destatis.de. Recuperado de https://www.destatis.de/DE/Publikationen/Thematisch/BildungFors chungKultur/Hochschulen/StudierendeHochschulenVorb21104101 48004.pdf;jsessionid=B99CB8D807AE638C 19906AFBA621B645.cae4 ?_blob=publicationFile 
Tiana Ferrer, A. (1991). La educación de adultos en el siglo XIX: Los primeros pasos hacia la constitución de un nuevo ámbito educativo. Revista de Educación, 294, 7-26.

Torres, R. M. (2003). Aprendizaje a lo largo de toda la vida: Un nuevo momento y una nueva oportunidad para el aprendizaje y la educación básica de adultos en el Sur. ASDI (Estocolmo), IIZ-DVV (Bonn) y IIEP-UNESCO (Paris).

UNESCO. (1976). Recomendación relativa al desarrollo de la educación de adultos. Naoribi. Recuperado de http://www.unesco.org/education/vie/confintea/nairob_s.pdf

UNESCO. (1995). La convección sobre los derechos del niño. París.

Wolter, A. (2012). From individual talent to institutional permeability: changing policies for non-traditional access routes in German higher education. En Slowey, M. \& Schuetze, H. (Eds.), Global perspectives on higher education and lifelong learners (pp. 43-59). Routeledge.

Zahl Der Studierenden. Deutsche Und Ausländische Studierende In Den 20 Am Stärksten Besuchten Hochschulen (2014). En Uni-muenster.de. Recuperado de http://www.unimuenster.de/wwu/statistik/lehre/studierendenzahl/studierendenza $\underline{\text { hl.html }}$

\section{Sobre la autora:}

\section{Alicia Sianes-Bautista}

Graduada en Pedagogía por la Universidad de Sevilla. Máster en Innovación e Investigación en Educación, especialidad en Historia de la Educación y Educación Comparada por la Universidad Nacional de Educación a Distancia. Actualmente trabaja como Pedagoga en el Centro de Psicología Infantil Ábaco (Alcalá de Guadaíra, Sevilla) y es Asistente Honoraria en el departamento de Teoría e Historia de la Educación y Pedagogía Social de la Universidad de Sevilla. 


\section{Para citar este artículo}

Sianes-Bautista, A. (2015). Aprendizaje a lo largo de la vida y educación superior de adultos en el ámbito universitario en Alemania. Origen y evolución histórica hasta la actualidad. Revista Fuentes, 17, Diciembre, pp. 89-112. [Fecha de consulta: dd/mm/aa]. doi.: doi: http://dx.doi.org/10.12795/revistafuentes.2015.i17.04. 